\title{
Role of MRCP in The Assessment of Patients Undergoing Cholecystectomy
} Marmar Essam Mahmoud Salama, Hossam Abd Elkader, Ahmed Mohamed Hussein

Radiology Department, Ain Shams Hospitals

Corresponding author: Marmar Salama, email: docmarmarsalama@gmail.com

\begin{abstract}
Aim of the work: this study aimed to assess if the Magnetic Resonance Cholangiopancreatography (MRCP) in patients undergoing cholecystectomy, changed the treatment plan or changed the operation method. Patients and method: this study was carried out in the Radiology Departments of Ain Shams University Hospitals. A total of 30 patients undergoing cholecystectomy were referred for preoperative MRCP. They were $20(66.7 \%)$ females and 10 (33.3\%) males. Their age was ranged from 20-60 years old. Results: Among the 30 cases, 21 cases had dilated common bile duct (CBD), 11 patients had CBD stones, 2 of which were silent, one case had silent CHD stone, one case had Mirizzi type I syndrome, 3 cases had trifurcation of the CHD, 4 cases had cystic duct anatomic variants and one case had duplicated GB. Conclusion: In candidate patients for cholecystectomy, routine preoperative MRCP is mandatory for confirmation of the already known pathologies, detection of other missed pathologies by US as well as detection of anatomical variants of biliary tract, these will help the surgeon for replanning and adjusting the surgical approach to preventing or at least decreasing the probability of post operative complications.
\end{abstract}

Keywords: MRCP, GB, Stones, cholecystectomy.

\section{INTRODUCTION}

Magnetic

resonance

cholangiopancreatography (MRCP) is well known to be a unique noninvasive technique for the diagnosis of biliary obstruction. It is well suited to provide the information required to plan the optimal therapeutic approach for these patients ${ }^{(1)}$. A preoperative diagnostic imaging procedure is essential for therapy in cholecystolithiasis. According to the S3-Guidelines of the German Society for General and Visceral Surgery only an ultrasound scan is needed before a cholecystectomy. But an anatomic variant of the bile ducts or choledocholithiasis is poorly shown by an ultrasound ${ }^{(2)}$.

Biliary anatomy and its common and uncommon variations are of considerable clinical significance when performing radiological interventions in hepatobiliary system, and laparoscopic cholecystectomy. Intrahepatic and extrahepatic bile duct variations are commonly seen. Normal biliary anatomy is seen in only $58 \%$ of the population. There are various techniques available for the visualization of biliary tree. Intravenous cholangiography often does not opacify the intra- and extrahepatic biliary tree and rarely allows a detailed visualization of the duct bifurcation. Endoscopic retrograde cholangiopancreatography (ERCP), although very accurate, is an invasive method for imaging the biliary tree. Intraoperative cholangiography is also highly accurate; however, it is an invasive procedure and its routine use remains controversial. Magnetic resonance cholangiopancreatography (MRCP) is an excellent non-invasive imaging technique for visualization of detailed biliary anatomy. High-resolution cross-sectional, two- dimensional (2D) and three-dimensional (3D) projection images provide excellent detailed anatomy which is comparable to ERCP and intraoperative Cholangiograms (3) Choledocholithiasis also may be asymptomatic; or symptomatic with potential complications including post-operative biliary leakage, recurrent biliary colics, cholangitis, and pancreatitis adding further to the burden of management in gallstone disease. Several studies were done to assess the importance of preoperative MRCP in reducing postoperative complications. However the role of routine use of pre-operative MRCP is still a matter of debate. So this randomized study was conducted to evaluate this role ${ }^{(4)}$.

\section{PATIENTS AND METHOD}

From January 2015 to January 2018, 30 randomized cases diagnosed as having acute or chronic cholecystitis and preparing for cholecystectomy, were referred from Tropical Medicine, General Surgery, and Clinical Oncology departments to the MRI unit at Diagnostic Radiology Department in the Ain Shams University Hospitals, for MRCP examination. The study was approved by the Ethics Board of Ain Shams University.

\section{Inclusion criteria}

- Both sexes were included

- All patients ranging from 20 to 60 years old Exclusion criteria

- Patients known to have contraindications for MRI, e.g. an implanted magnetic device, pacemakers or claustrophobia.

- Any patient with symptoms suggesting pancreatitis or cholangitis 


\section{Procedure}

Patients were subjected to:

1- Full history taking.

2- Abdominals U/S, liver functions (including SGOT, SGPT, PT time and concentration, total, direct bilirubin, and alkaline phosphatase were checked.

3- All patients underwent MRCP examination on 1.5 T machine (Achieva, Philips medical system, Eindhoven, Netherlands).

\section{Patient preparation}

- Explanation of the study to patients and obtaining consent.

- Fasting for 4 hours prior to the examination to promote gall bladder filling, gastric emptying, reduce unwanted fluid signal from the intestine and to reducing the intestinal motility. No oral contrast agents or antiperistaltic agents were administrated.

- Patients were supine with head first on the examination couch.

\section{Procedure duration: 15 -20 minutes. Method:}

Axial T2 fast spin-echo (FSE) MR examination of the upper abdomen was performed first, to optimally localize the biliary system and to plan the MRCP.MRCP was performed with two different techniques:

I- Respiratory Triggered, Three Dimensional (3D) MRCP with MIP reconstruction: The following parameters were used:

- Repetition time (msec) /echo time (msec): 1800/350.

- Echo train length: 100

- Flip angle: 90

- Number of excitation (NEX): 2

- Field of view: $300 \mathrm{~mm}$

- Matrix: 78 x 512

- Section thickness: $3 \mathrm{~mm}$ with $1.5 \mathrm{~mm}$ overlap.

- Number of slices: 50

- Scan time: ranging from 1.23 to 3 minutes.

Multi-section MRCP was performed with respiratory triggering in order to reduce respiratory motion artifacts. With use of respiratory triggering, the data were acquired between successive respiratory cycles (i.e. from the end of expiration to the beginning of the following inspiration). The use of respiratory triggering increased the actual scan time depending on the patient's breathing pattern and respiratory rate. No specific instructions were given to the patients regarding respiration. Coronal images were obtained in right anterior oblique plane parallel to the longitudinal axis of the biliary tree. The obtained source images (about 40 images) were compressed and reconstructed with MIP algorithm to create a 3D cholangiographic images that could be viewed from multiple directions. Reconstruction was performed, 9 to 12 oblique images were obtained with frontal to lateral reconstruction at $12^{\circ}-15^{\circ}$ intervals.

II- Breath Hold, Two Dimensional (2D), Single shot MRCP, single slice technique: A single-shot turbo spin-echo sequence was used. Imaging parameters were as follows:

- Repetition time $(\mathrm{msec}) / e c h o$ time $(\mathrm{msec})=$ 8,000/900

- $\quad$ Echo train length: 256

- Flip angle: 90

- Number of excitation (NEX): 1

- Field of view: $370 \mathrm{~mm}$

- Matrix: 205 x 256

- Section thickness: 40-50 mm

- Scan time: 8 seconds which permits breathhold scanning.

The thick coronal as well as coronal oblique slabs were obtained at different angles from a horizontal plane pass through the head of pancreas to ensure including the distal part of the pancreaticobiliary tract in all images. The first slab was acquired at the direct coronal plane and about 4-5 slabs with different degrees of obliquity were acquired on either side of the mid horizontal plane. Cholangiograms like images were acquired and no further post processing was required.

In all the examined cases MRCP images were evaluated for:

- Anatomical variations along gall bladder as well as pancreaticobiliary tree.

- Calcular and / or non calcular cholecystitis.

- The dilatation of the intrahepatic and extrahepatic bile ducts as well as the pancreatic duct.

- The level of obstruction, the morphological aspect at the obstruction site.

- Any additional information provided by the axial TIWI and T2WI (tumors and their extension, the presence of lymphadenopathies or metastases).

\section{Statistical analysis}

The statistical data were analyzed using Chisquared test. $\quad P<0.05$ was considered statistically significant.

\section{RESULTS}

A total of 30 patients were presented with diagnosis of cholecystitis. They were 20 (66.7\%) females and 10 (33.3\%) males.

Age: Their age ranged from 20 years 60 years old. 
Table 1. Clinical presentation of the studied group

\begin{tabular}{|l|l|c|c|}
\hline \multicolumn{2}{|c|}{ Presenting symptoms } & No. & $\%$ \\
\hline Abdominal & No & 0 & $0.0 \%$ \\
pain & Yes & 30 & $100.0 \%$ \\
\hline \multirow{2}{*}{ Jaundice } & No & 9 & $30.0 \%$ \\
& Yes & 21 & $70.0 \%$ \\
\hline \multirow{2}{*}{ Biliary colic } & No & 23 & $76.7 \%$ \\
& Yes & 7 & $23.3 \%$ \\
\hline
\end{tabular}

Table 2. previous lab results of the study group

\begin{tabular}{|l|c|c|}
\hline $\begin{array}{c}\text { Elevated liver } \\
\text { enzymes and } \\
\text { bilirubin }\end{array}$ & No. & $\%$ \\
\hline No & 9 & $30.0 \%$ \\
Yes & 21 & $70.0 \%$ \\
\hline
\end{tabular}

Table 3. Ultrasound findings in study group:

\begin{tabular}{|c|c|c|c|}
\hline \multicolumn{2}{|c|}{ Us findings } & No. & $\%$ \\
\hline \multirow{2}{*}{$\begin{array}{l}\text { Non calcular } \\
\text { cholecystitis }\end{array}$} & No & 28 & $93.3 \%$ \\
\hline & Yes & 2 & $6.7 \%$ \\
\hline \multirow{2}{*}{$\begin{array}{l}\text { Calcular } \\
\text { cholecystitis }\end{array}$} & No & 3 & $10.0 \%$ \\
\hline & Yes & 27 & $90.0 \%$ \\
\hline \multirow{2}{*}{$\begin{array}{l}\text { Intrahepatic } \\
\text { biliary radicle } \\
\text { dilatation } \\
\text { (IHBRD) }\end{array}$} & No & 22 & $73.3 \%$ \\
\hline & Yes & 8 & $26.7 \%$ \\
\hline \multirow{2}{*}{$\mathrm{CBD}$ dilatation } & No & 17 & $56.7 \%$ \\
\hline & Yes & 13 & $43.3 \%$ \\
\hline
\end{tabular}

Table 4. MRCP gall bladder pathologies and anatomical variations

\begin{tabular}{|l|l|c|c|}
\hline \multicolumn{2}{|c|}{$\begin{array}{c}\text { MRCP gallbladder } \\
\text { pathologies }\end{array}$} & No. & $\%$ \\
\hline $\begin{array}{l}\text { Non calcular } \\
\text { cholecystitis }\end{array}$ & No & 27 & $90.0 \%$ \\
\hline Calcular & 3 & $10.0 \%$ \\
\hline cholecystitis & No & 4 & $13.3 \%$ \\
\hline \multirow{2}{*}{ Adenomyomatosis } & No & 26 & $86.7 \%$ \\
\hline \multirow{2}{*}{ Gall bladder mass } & Yes & 1 & $96.7 \%$ \\
& No & 30 & $100.0 \%$ \\
\hline \multirow{2}{*}{ GB variant } & Nos & 0 & $0.0 \%$ \\
\hline & Yes & 1 & $96.7 \%$ \\
& & $3.3 \%$ \\
\hline
\end{tabular}

Table 5. MRCP cystic duct pathologies and anatomical variations

\begin{tabular}{|l|l|c|c|}
\hline \multicolumn{2}{|c|}{$\begin{array}{c}\text { MRCP cystic duct } \\
\text { pathologies }\end{array}$} & No. & $\%$ \\
\hline \multirow{2}{*}{ Dilated } & No & 25 & $83.3 \%$ \\
& Yes & 5 & $16.7 \%$ \\
\hline Containing & No & 24 & $80.0 \%$ \\
stone/s & Yes & 6 & $20.0 \%$ \\
\hline Cystic duct & No & 26 & $86.7 \%$ \\
variant & Yes & 4 & $13.3 \%$ \\
\hline
\end{tabular}

Table 6. MRCP IHBR pathologies and anatomical variations:

\begin{tabular}{|l|l|r|c|}
\hline \multicolumn{2}{|c|}{ MRCP IHBR pathologies } & No. & $\%$ \\
\hline \multirow{2}{*}{ Dilated } & No & 10 & $33.3 \%$ \\
& Yes & 20 & $66.7 \%$ \\
\hline Dilated with & No & 30 & $100.0 \%$ \\
stone & Yes & 0 & $0.0 \%$ \\
\hline other & No & 30 & $100.0 \%$ \\
pathologies & Yes & 0 & $0.0 \%$ \\
\hline \multirow{2}{*}{ IHBR variants } & No & 27 & $90.0 \%$ \\
& Yes & 3 & $10.0 \%$ \\
\hline
\end{tabular}

Table. 7. MRCP CHD pathologies

\begin{tabular}{|l|l|r|c|}
\hline \multicolumn{2}{|c|}{ MRCP CHD pathologies } & No. & $\%$ \\
\hline \multirow{2}{*}{ Dilated } & No & 12 & $40.0 \%$ \\
& Yes & 18 & $60.0 \%$ \\
\hline Dilated with & No & 29 & $96.7 \%$ \\
stone & Yes & 1 & $3.3 \%$ \\
\hline \multirow{2}{*}{ Silent stone } & No & 29 & $96.7 \%$ \\
& Yes & 1 & $3.3 \%$ \\
\hline \multirow{2}{*}{ Other } & No & 28 & $93.3 \%$ \\
pathologies & Mirizzi & 1 & $3.3 \%$ \\
& type I & 1 & $3.3 \%$ \\
\hline
\end{tabular}

Table. 8. MRCP CBD pathologies

\begin{tabular}{|c|c|c|c|}
\hline \multicolumn{2}{|c|}{ MRCP CBD pathologies } & No. & $\%$ \\
\hline \multirow{2}{*}{ Dilated } & No & 9 & $30.0 \%$ \\
\hline & Yes & 21 & $70.0 \%$ \\
\hline \multirow{2}{*}{$\begin{array}{l}\text { Dilated } \\
\text { with stone }\end{array}$} & No & 19 & $63.3 \%$ \\
\hline & Yes & 11 & $36.7 \%$ \\
\hline \multirow{2}{*}{$\begin{array}{l}\begin{array}{l}\text { Silent } \\
\text { stone }\end{array} \\
\end{array}$} & No & 28 & $93.3 \%$ \\
\hline & Yes & 2 & $6.7 \%$ \\
\hline \multirow{3}{*}{$\begin{array}{l}\text { Other } \\
\text { pathologies }\end{array}$} & No & 25 & $83.3 \%$ \\
\hline & $\begin{array}{l}\text { Choledochal } \\
\text { type VI }\end{array}$ & 1 & $3.3 \%$ \\
\hline & Stricture & 4 & $13.3 \%$ \\
\hline CBD & No & 30 & $100.0 \%$ \\
\hline variants & Yes & 0 & $0.0 \%$ \\
\hline
\end{tabular}

Table.9. MRCP pancreatic duct pathologies and surgical strategy of management affection:

\begin{tabular}{|l|l|r|r|}
\hline \multicolumn{2}{|l|}{} & No. & $\%$ \\
\hline $\begin{array}{l}\text { MRCP } \\
\text { pancreatic duct } \\
\text { pathologies } \\
\text { (Dilated) }\end{array}$ & No & 29 & $96.7 \%$ \\
\hline $\begin{array}{l}\text { Post MRCP } \\
\text { changed } \\
\text { surgical } \\
\text { strategy of } \\
\text { management }\end{array}$ & No & 4 & $3.3 \%$ \\
\hline
\end{tabular}


Table 10. Relation between US and MRCP in detecting variants

\begin{tabular}{|l|l|c|c|}
\hline \multicolumn{2}{|c|}{} & Count & $\%$ \\
\hline US & Normal & 30 & $100.0 \%$ \\
\cline { 2 - 4 } variants & detect variants & 0 & $0.0 \%$ \\
\hline MRCP & Normal & 22 & $73.3 \%$ \\
\cline { 2 - 4 } variants & detect variants & 8 & $26.7 \%$ \\
\hline
\end{tabular}

\begin{tabular}{|l|l|c|c|}
\hline \multicolumn{2}{|c|}{} & Count & $\%$ \\
\hline \multirow{2}{*}{$\begin{array}{l}\text { US } \\
\text { pathologies }\end{array}$} & Normal & 15 & $50.0 \%$ \\
\cline { 2 - 4 } $\begin{array}{l}\text { MRCP } \\
\text { pathologies }\end{array}$ & Netect pathology & 15 & $50.0 \%$ \\
\cline { 2 - 4 } & Dermal & 4 & $13.3 \%$ \\
\hline
\end{tabular}

Table 11. Relation between US and MRCP in detecting pathologies in general:

Using Chi-squared test in analyzing the relation between MRCP and US In detecting pathologies in general the The P-value of this relation is 0.025 which is statistically significant (P-values less than 0.05 were considered as statistically significant).

\section{CASES}

Case 1: Female patient 58 years old presented with epigastric pain radiating to the back. US revealed: Acute calcular cholecystitis with dilated CBD. MRCP revealed: Slight ectasia of extrahepatic CBD which shows no distal stones or obstructing masses. The gall bladder is distended with stones inside with cystic duct is seen joining CHD at the distance of about $2 \mathrm{~cm}$ from the confluence of hepatic duct. Prominent ventral pancreatic duct opens at minor duodenal papilla and is seen draining suggested accessory small gall bladder (double gall bladder) which in turns shows no stones inside (Fig. 1).

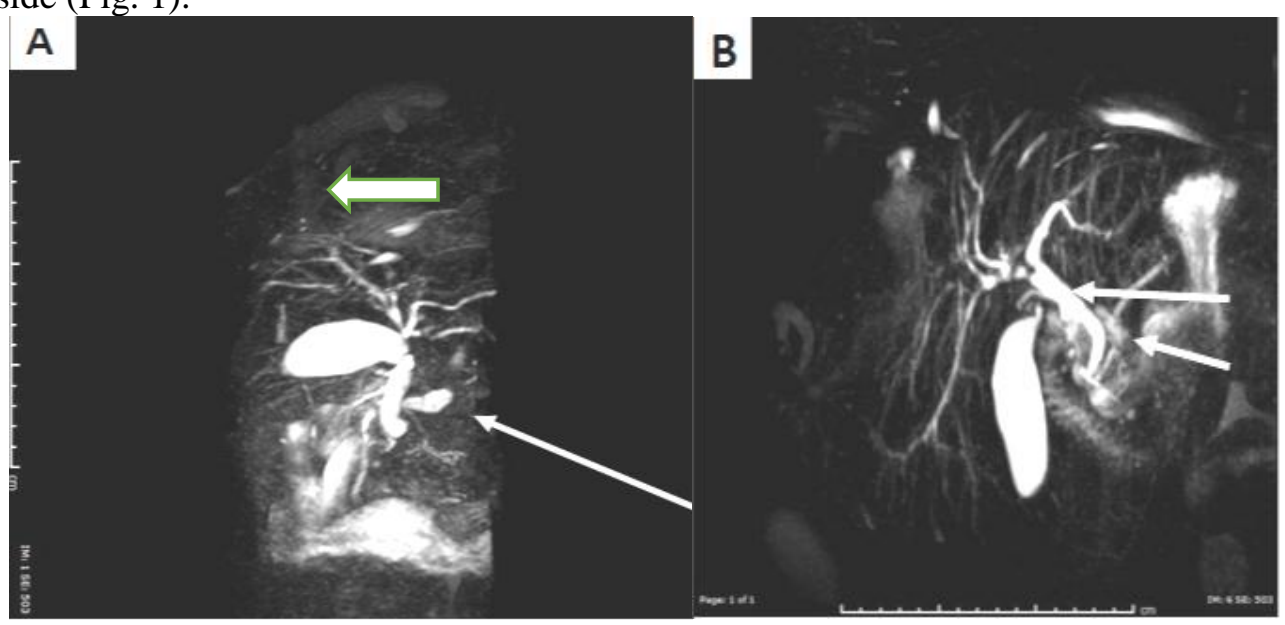

Fig. (1) (A) and (B) 3D MRCP images show double gall bladder with minimal biliary stasis (stone passer), (A) arrow is pointing to the accessory gall bladder, (B) the long arrow is pointing to the ecstatic $\mathrm{CBD}$, and the short arrow is pointing to the accessory gall bladder with prominent pancreatic duct.

Case 2: Female patient 49 years old presented with jaundice and right hypochondrial pain.US revealed: Calcular cholecystitis. MRCP revealed: Gall bladder is well distended with few stones seen inside of average $3 \mathrm{~mm}$ with one of them seen at the neck of the gall bladder. Low lying insertion of the cystic duct, seen inserted at the distal part of CBD near its insertion in the ampulla of Vater (Fig. 2).

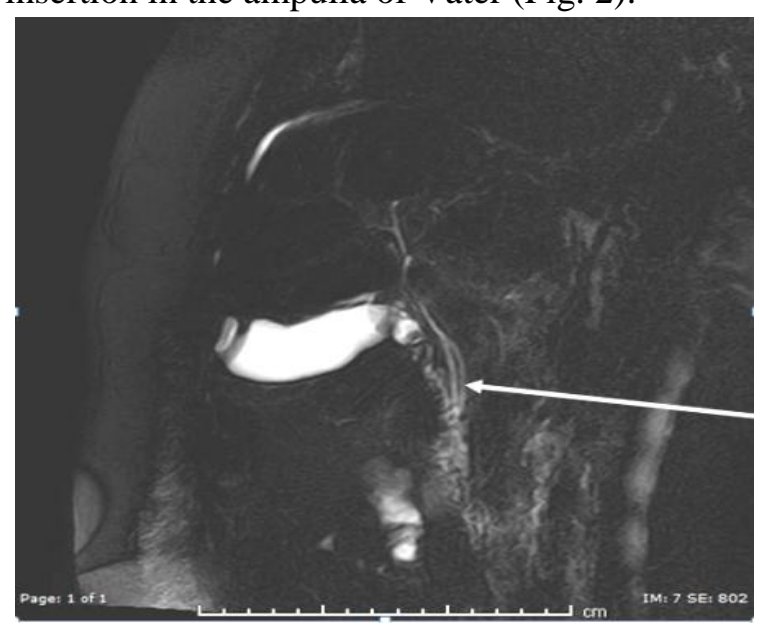


Fig. (2): 3D MRCP image (source images MIP reformatted thin cuts). (Short arrow) is pointing to gall bladder neck stone, (long arrow) is pointing to parallel low lying course of the cystic duct to the CBD.

Case 3: Male patient 55 years old presented with jaundice abdominal pain. US revealed: Calcular cholecystitis. MRCP revealed: a large $2 \mathrm{~cm}$ stone in the cystic duct with gall bladder seen partially contracted with thick wall and tiny stone inside likely chronic cholecystitis. Slightly prominent CHD and CBD which are seen measuring $8 \mathrm{~mm}$. No biliary dilatation, strictures or filling defects with minimal dilatation of the intrahepatic biliary radicals (Fig. 3).

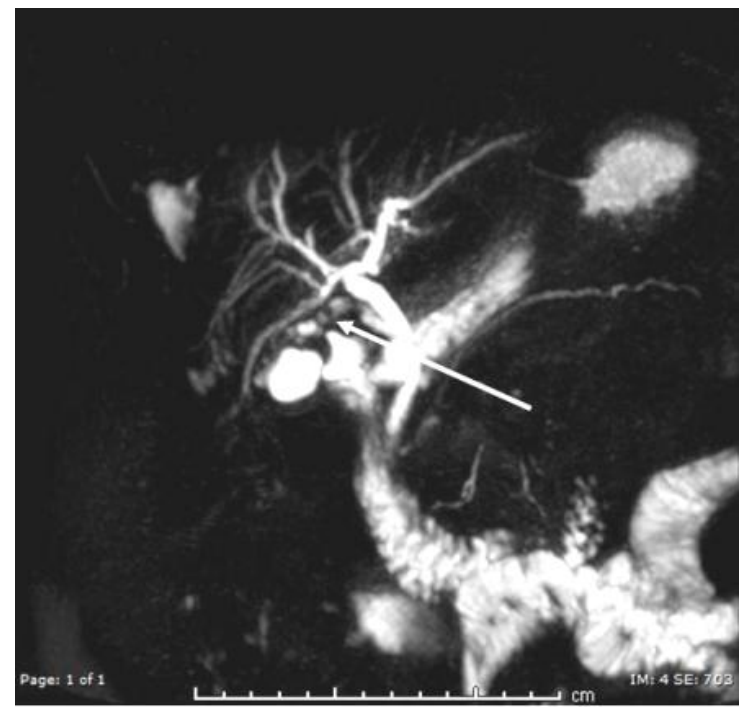

Fig (3): 3D MRCP image showing prominent intra and extrahepatic biliary radicals with (arrow) pointing to cystic duct stone (signal void).

\section{DISCUSSION}

Chowbey et al. ${ }^{(5)}$ stated that laparoscopic cholecystectomy is an established operation for symptomatic gall stone disease. It provides total relief of pre-surgical symptoms in up to $85 \%$ of patients. However about $5 \%$ of patients may experience severe episodes of upper abdominal pain similar to those that they had prior to cholecystectomy. These symptoms may be due to biliary stricture, retained / recurrent biliary calculi, stenosis or dyskinesia of sphincter of Oddi, cystic neuroma, remnant gall bladder / cystic duct stump calculi etc. and are together grouped as post cholecystectomy syndrome. Jonson et al. ${ }^{(6)}$ stated that several reports have proposed that a cystic duct remnant $>1 \mathrm{~cm}$ in length after cholecystectomy may be responsible, at least in part, for postcholecystectomy syndrome. Nagral ${ }^{(7)}$ reported that anatomical facts are of relevance to the performance of a safe cholecystectomy. Misinterpretation of normal anatomy and anatomical variations contribute to the occurrence of major postoperative complications like biliary injuries following a cholecystectomy, the incidence being higher with laparoscopic cholecystectomy. A look at the basic anatomy is therefore important for biliary and minimally invasive surgeons. This includes normal anatomy and variations of the biliary apparatus as well as the arterial supply to the gallbladder. Miller et al. ${ }^{(8)}$ stated that choledocholithiasis is a common problem seen in as many as $6-12 \%$ of patients undergoing cholecystectomy. Common bile duct stones may be asymptomatic. Although common bile duct (CBD) stones may be silent but it can often lead to biliary colic, cholangitis, jaundice, or pancreatitis. There are various techniques available for the visualization of biliary tree Costi et al. ${ }^{(9)}$ reported that ERCP after having been widely used for CBD stones diagnosis in the late eighties/nineties. ERCP is nowadays being progressively abandoned as a diagnostic tool for patients with moderate to intermediate risk of carrying CBD stones, as most cholangiograms result as being normal and ERCP is not costeffective. Compared to EUS and MRCP, accuracy of ERCP is suboptimal, being reduced in the case of CBD dilation and small CBDS stones. Moreover, ERCP not only involves X-ray exposure and the intrinsic invasiveness of endoscopy, but it also has non-negligible procedure-related morbidity/mortality, with a $2 \%-11 \%$ acute pancreatitis rate. Moreover, once ERCP is performed, endoscopic sphincterotomy is frequently associated regardless of CBD stones presence, both for the risk of a post-ERC ascending cholangitis and for the possible false negatives of ERCP itself. 
Duncan and Riall ${ }^{(10)}$ stated that MRI and MRCP are useful in identifying CBD stones and delineating pancreatic and biliary tract anatomy. CBD stones, if identified on MRCP, cannot be removed, necessitating additional therapeutic procedures such as ERCP or common bile duct exploration. Conversely, if MRCP is negative, the complications associated with these more invasive procedures can be avoided. The current study was aiming to clarify the importance of MRCP as a preoperative imaging modality in candidate patients for cholecystectomy, to know the anatomical pattern of their intra as well as extra-hepatic bile ducts and also to detect any silent CBD pathologies missed to be diagnosed by primary imaging techniques as US. Jaba et al. ${ }^{(11)}$ stated that, the incidence of duplicated gall bladder was ranged 1:3000 to 1:12000; while due to limited number of cases in current study, the incidence of duplicated gall bladder was higher $(3.3 \%)$ and was seen in one case. In current study, there was no case $(0 \%)$ detected with Phrygian cap, while Jaba et $\boldsymbol{a l} .^{(11)}$ reported Phrygian cap in (6\%) of their study. This inconsistency is due to limited number of cases in our study. Turner and Fulcher ${ }^{(12)}$ found that, (10.6 $\%)$ of their study group with parallel course low inserted cystic duct to CHD, (18.4\%) with medially low inserted cystic duct to CHD and $(71 \%)$ with normal laterally inserted cystic duct to the middle third of CHD; while in the current study, parallel low inserted cystic duct were detected in two cases $(6.6 \%)$, medially low inserted cystic duct were detected in two cases $(6.6 \%)$ and normal laterally inserted cystic duct to the middle third of CHD were detected in twenty six cases (86.8\%). Blumgart ${ }^{(13)}$ reported that, the incidence of normal anatomical pattern of IHBR were detected in (57\%) of his cases, trifurcation pattern were detected in (12\%), the right posterior duct drained to the left hepatic duct were detected in $(21 \%)$, the right posterior duct drained directly to the common hepatic duct were detected in $(4 \%)$, segmental (segment VI) intrahepatic biliary duct drianed to the cystic duct were detected in $(2 \%)$ and $(4 \%)$ were related to the right anterior ducts variants. By observing the anatomy of intrahepatic bile ducts, the current study found that, twenty seven $(90 \%)$ cases with normal anatomical pattern of IHBR as right anterior and posterior sectorial ducts were joined to form the right hepatic duct that joined to left hepatic duct to form CHD, three cases $(10 \%)$ with trifurcation of the right anterior duct with the left hepatic duct, Unfortunately, the current study did not find anatomical variants related to the right anterior or posterior intrahepatic bile ducts. In the present study, the gall bladder stones were detected in twenty six cases (86.7\%). Thirteen cases (43.3 $\%)$ also had CBD stones while Stuart et al. ${ }^{(14)}$ found that, the incidence of CBD stones among the patients with gall bladder stones was $12 \%$. Meena et al. ${ }^{(15)}$ found that, (24\%) of their study group had extrahepatic bile duct stones. In the current study, sixteen cases (53\%) of our study group had extrahepatic bile duct stones. Fourteen of them were also had gallbladder stones and the remaining cases had no evidence of gallbladder stones. In the present study, two (12.5\%) of sixteen cases with extrahepatic bile duct stones were asymptomatic (silent) stones and were reported as normal cases by US, while Topal et al. ${ }^{(16)}$ stated that, the incidence of asymptomatic (silent) extrahepatic bile duct stones was 5\%. Meena et al. ${ }^{(15)}$ reported that, $(28 \%)$ of their cases had malignant biliary obstruction, more than half of them were caused by cholangiocarcinoma while the rest were caused by pancreatic and duodenal causes. In the current study, none of the cases (0\%) of our study group had malignant biliary obstruction. In current study also, none of the cases $(0 \%)$ had primary sclerosing cholangitis were detected. Katabathina et al. ${ }^{(17)}$ reported that, the incidence of primary sclerosing cholangitis in their study was (3.8\%). In the present study, the US couldn't detect anatomical variants in any case $(0 \%)$, while MRCP had detected anatomical variants in eight cases $(26.7 \%)$ out of thirty. On the other hand, the US in current study had detected different pathologies in fifteen cases $(50 \%)$ out of thirty cases, while MRCP had detected different pathologies in twenty six $(86.7 \%)$ out of thirty cases, which means that; eleven $(42.3 \%)$ of these twenty six cases were not detected by US, which is statistically significant difference $(\mathrm{P}$ value $=0.025)$. Ankur et al. ${ }^{(18)}$, Sureka et al. ${ }^{(3)}$, and Yun-Hua et al. ${ }^{(19)}$ stated that, routine preoperative MRCP examination is recommended for patients candidate for cholecystectomy to prevent or at least decreasing the probability of post operative complications. At the end of current study, we are totally agreeing with them, as there were two cases with asymptomatic (silent) extrahepatic bile duct stones were reported as normal cases by US as well as eight cases with different biliary tract anatomical variants were not detected by US; however, these findings were detected by MRCP, which means that, MRCP has added important information that modified the surgical planning in these cases. Finally: we conclude that, in the patients candidate for cholecystectomy, routine preoperative MRCP is mandatory for confirmation of the already known pathologies, detection of other missed pathologies by US as well 
as detection of anatomical variants of biliary tract, these will help the surgeon for replanning and adjusting the surgical approach to preventing or at least decreasing the probability of postoperative complications.

\section{REFERENCES}

1. Kalra M, Sahani D, Ahmad A, Saini S(2002): The role of magnetic resonance cholangiopancreatography in patients with suspected biliary obstruction. Curr Gastroenterol Rep., 4(2):160-6.

2. Schmidt R, Tannheimer M, Danz B, Benesch S, Geue $\mathbf{R}(\mathbf{2 0 1 2})$ : Clinical relevance of a routinely performed magnetic resonance cholangiopancreatography (MRCP) prior to cholecystectomy. Zentralbl Chir. ,137(6):541-8

3. Sureka B, Bansal K, Patidar Y, Arora A(2016): Magnetic resonance cholangiographic evaluation of intrahepatic and extrahepatic bile duct variations. Indian J Radiol Imaging, 26(1):22-32.

4. Bahram M, Gaballa G(2010): The value of preoperative magnetic resonance cholangiopancreatography (MRCP) in management of patients with gall stones. Int J Surg., 8(5):342-5.

5. Chowbey P, Sharma A, Goswami A, Afaque Y, Najma K, Baijal M, Soni V, Khullar R (2015): Residual gallbladder stones after cholecystectomy: A literature review. Journal of Minimal Access Surgery, 11(4):223.

6. Jonson G, Nilsson DM, Nilsson T(1991): Cystic duct remnants and biliary symptoms after cholecystectomy. A randomised comparison of two operative techniques. Eur J Surg., 157:583-6.

7. Nagral S(2005): Anatomy relevant to cholecystectomy. Journal of Minimal Access Surgery, 1(2): 53.

8. Miller FH, Hwang CM, Gabriel H, Goodhartz LA, Omar AJ, Parsons WG(2003): Contrast-enhanced helical CT of choledocholithiasis. American Journal of Roentgenology, 181(1):125-30.

9. Costi R, Gnocchi A, Di mario F, Sarli L(2014): Diagnosis and management of choledocholithiasis in the golden age of imaging, endoscopy and laparoscopy. World J Gastroenterol., 20(37):13382-401.

10. Duncan CB, Riall TS(2012): Evidence-based current surgical practice: calculous gallbladder disease. J Gastrointest Surg., 16(11):2011-25.

11. Jaba R, Satyam K, Shilpi J, Rashmi G, Mukesh S and Prabhat G(2012): Variations In The External Morphology Of Gallbladder. J. Anat. Soc. India, 61(1):9-12.

12. Turner MA and Fulcher AS(2001): The cystic duct: normal anatomy and disease processes. Radiographics, 21(1):3-22.

13. Blumgart $\mathbf{L H}(\mathbf{2 0 0 0})$ : Surgery of the Liver and biliary tract, 3rd ed., P.19.

14. Stuart M, Sukhpal $S$ and Iain $\mathbf{P ( 2 0 0 7 ) : ~ S e l e c t i v e ~}$ $\mathrm{MRCP}$ in the management of suspected common bile duct stones. HPB (Oxford), 9(2):125-130.

15. Meena S, Sunita $P$, Vivek $B$ and Pradeep G(2015): Role of MRCP in Differentiation of Benign and Malignant Causes of Biliary Obstruction. Journal of clinical and Diagnostic Research, 9(11):TC08-TC12.

16. Topal B, Van de Moortel M, Fieuws S et al.(2003): The value of magnetic resonance cholangiopancreatography in predicting common bile duct stones in patients with gallstone disease. $\mathrm{Br} \mathrm{J}$ Surg., 90:42-47.

17. Katabathina VS, Dasyam AK, Dasyam N, Hosseinzadeh K(2014): Adult Bile duct strictures: Role of MR Immaging and MR Cholangiopancreatography in Characterization. Radio Graphics, 34:565-86.

18. Ankur $M$, Arun $K$, Devendra $K$ and Sanjeev S(2013): The value of Magnetic Resonance Cholangio-Pancreatography (MRCP) in the Detection of Choledocholithiasis. J Clin Diagn Res., 7(9):19411945.

19. Yun-Hua W, Zhi-Su L, Rekia M, Zhong-Li A, Quan S, Gassimou Ba, Qun Q, and Cong-Qing J(2008): Anatomical variations of the cystic duct: Two case reports. World J Gastroenterol., 14(1):155157. 